\title{
Cdc6 cooperates with c-Myc to promote genome instability and epithelial to mesenchymal transition (EMT) in zebrafish
}

\author{
Ching-Hung Chen ${ }^{1}$, Dar-Shong Lin ${ }^{2,3,4,5}$, Chieh-Wen Cheng ${ }^{6}$, Chun-Ju Lin ${ }^{1}$, Yu-Kang \\ Lo $^{6}$, Chueh-Chuan Yen ${ }^{7,8,9}$, Alan Yueh-Luen Lee ${ }^{6}$ and Chung-Der Hsiao ${ }^{1,10}$ \\ ${ }^{1}$ Department of Bioscience Technology, Chung Yuan Christian University, Chung-Li, Taiwan \\ 2 Department of Pediatrics, Mackay Memorial Hospital, Taipei, Taiwan \\ ${ }^{3}$ Department of Medical Research, Mackay Memorial Hospital, Taipei, Taiwan \\ ${ }^{4}$ Mackay Junior College of Medicine, Nursing, and Management, Taipei, Taiwan \\ ${ }^{5}$ Mackay Medical College, Taipei, Taiwan \\ ${ }^{6}$ National Institute of Cancer Research, National Health Research Institutes, Miaoli, Taiwan \\ 7 Division of Hematology \& Oncology, Department of Medicine, Taipei Veterans General Hospital, Taipei, Taiwan \\ ${ }^{8}$ National Yang-Ming University School of Medicine, Taipei, Taiwan \\ ${ }^{9}$ Therapeutical and Research Center of Musculoskeletal Tumor, Taipei Veterans General Hospital, Taipei, Taiwan \\ ${ }^{10}$ Center for Nanotechnology, Chung Yuan Christian University, Chung-Li, Taiwan \\ Correspondence to: Alan Yueh-Luen Lee, email: alanylee@nhri.org.tw
}

Chung-Der Hsiao, email: cdhsiao@cycu.edu.tw

Keywords: Cdc6/ c-Myc/ zebrafish /genomic instability/ epithelial to mesenchymal transition

Received: May 24, $2014 \quad$ Accepted: July 11, $2014 \quad$ Published: July 11, 2014

This is an open-access article distributed under the terms of the Creative Commons Attribution License, which permits unrestricted use, distribution, and reproduction in any medium, provided the original author and source are credited.

\section{ABSTRACT}

Aberration in DNA replication is a major cause to genome instability that is a hallmark of cancer cells. Cell division cycle 6 (Cdc6) and c-Myc have a critical role in the initiation of DNA replication. However, whether their interaction induces epithelial-mesenchymal transition (EMT) and promotes tumorigenesis in in vivo animal model remains unclear. Since using zebrafish as a cancer model has been restricted by the late onset of tumorigenesis and extreme difficulty in transformation on skin, we tried to establish a novel non-melanoma skin model in zebrafish to study their role in tumorigenesis. A stable transgenic zebrafish was created by using tol 2 transposon, in which cdc6 and c-myc were co-overexpressed in epidermis driven by a skin-specific krt4 promoter. Intriguingly, co-overexpression of cdc6 and c-myc in transgenic zebrafish skin triggered tumor-like transformation, apoptosis attenuation, genomic instability, and EMT, hallmarks of malignant tumorigenesis. Our findings and other characteristics of zebrafish, including optical clarity and small molecule treatment, provide the future utility of this model for easy and non-invasive detection and for identification of new anti-cancer drug.

\section{INTRODUCTION}

DNA replication is initiated by coordinated binding and highly controlled activation of the prereplicative complex (pre-RC). The pre-RC is called a replication licensing machinery including two replication licensing factors, Cdt1 and Cdc6 that are recruited during G1-S transition on the origin recognition complexes and promote the loading of mini-chromosome maintenance
(MCM) complex on chromatin [1, 2]. Deregulation of normal replication mechanism can create a cellular environment, termed replication stress [3], prone to genomic instability that is a hallmark of cancer [4]. Indeed, high levels of Cdc6 and Cdt1 have been observed in various types of cancer [5-8]. Cdc6 has been reported to interact with c-Myc that increases the replication activity with subsequent DNA damage [9]. However, the ability of Cdt1 and Cdc6 overexpression leads to rereplication, 
providing seeds for chromosome instability, has been recently challenged [10]. In addition, overexpression of Cdc6 induced by Ras or Mos oncogenes causes replication stress that activates DNA damage response (DDR)-mediated senescence $[11,12]$. In addition to the licensing factor mechanism, Cdc6 interacts with c-Myc to repress E-box-dependent transcription by interfering with the formation of c-Myc/Max complex [13]; it exert its oncogenic activity by repressing the INK4/ARF [7] and $C D H 1$ locus [14] that encodes E-cadherin, a marker of epithelial to mesenchymal transition (EMT). However, the issues of whether their interaction induces EMT and whether the interaction promotes cancer development in in vivo animal model remain obscure.

The zebrafish has become a good lower vertebrate model for studying carcinogenesis in recent years. Compared to other higher vertebrate models, zebrafish model advantages its rapid development, fecundity, amenability to both forward and reverse genetic strategies, body transparency, and suitability to in vivo imaging and chemical screening. Importantly, tumor in zebrafish resembles various types of human tumors at either the histological level [15] or cancer characteristics such as genomic instability, neoplastic cell transformation, and oncogene activation [16, 17]. For instance, several lossof-function of tumor suppressors, such as tp53 [18], PTEN [19] and APC [20], or gain-of-function of oncogenes, c-myc [21], Akt2 [22], and K-ras [23], have been established in zebrafish, which showed similar phenotypes to human cancer.

Although great advances have been achieved in zebrafish cancer model, several obstacles still need to be overcome in order to develop zebrafish as a promising and complementary cancer model. First, the onset of tumor transformation is quite late and usually occurs during juvenile to adult stages. For example, the first appearance of liver cancer in zebrafish is around 3 to 4 weeks post-fertilization after deregulated activation of oncogenes [24]. Second, some tissues like skin proceed oncogenic transformation with extreme difficulty. Third, since the body transparency at adult stage is reduced, it is hard to detect tumor formation and metastasis at adult stage by appearance. Therefore, how to induce the early onset of cancer formation on skin and develop an easy, non-invasive detection methodology are two key issues to the establishment of zebrafish cancer model.

In this study, we aimed to create a novel nonmelanoma skin cancer model in zebrafish by cooverexpression of Cdc6 and c-Myc to study their oncogenic mechanisms through the observation on an early onset and visible transformation on the outmost skin layer. To generate the transgenic zebrafish model, we used tol 2 transposon tool to overexpress cdc6 and c-myc in epidermis driven by a skin-specific krt4 promoter. Here we successfully created the stable zebrafish transgenics carrying cdc6 and c-myc. Furthermore, we investigated the underlying mechanism of cellular transformation triggered by co-overexpression of Cdc6 and c-Myc using a transgenic zebrafish skin model, which is involved in accelerated cell proliferation, apoptosis attenuation, genomic instability, and EMT.

\section{RESULTS}

\section{Single overexpression of cde6 or c-myc is not sufficient to oncogenic transformation of zebrafish skin}

To address the role of interaction between Cdc6 and c-Myc in tumorigenesis in vivo, we chose zebrafish as a model to study multiple-oncogenic transformation on the zebrafish skin. To determine the gain-of-function of Cdc6 and/or c-Myc on epidermal transformation in zebrafish, we constitutively overexpressed human cdc6 (hcdc6) and mouse c-myc T58A (m.c-myc T58A) genes in epidermis under the control of keratin4 (krt4) promoter (Fig. S1A). The cmlc2-EGFP-pA mini-cassette encodes a cytoplasmic-targeted EGFP, being a reporter for identification of transgenics. We successfully isolated four lines carrying cdc6 (germ-line transmission rate $=7 \%)$ and 28 lines carrying c-myc (61\%). Among all lines, $\operatorname{Tg}(\mathrm{krt} 4 \text { :hcdc6 })^{\mathrm{cy} 24}$ and $\operatorname{Tg}(\mathrm{krt} 4 \text { : m.c-myc T58A) })^{\text {cy21 }}$ displayed the most strongest green fluorescence in the heart and were kept for the following experiments. We used genotyping to identify stable krt4-cdc6 or c-myc expression according to positive genomic PCR results in the F1 transgenic progeny (Fig. S1A).

However, we were unable to observe any oncogenic transformation on skin when either cdc6 or c-myc transgene was overexpressed. To clarify this observation, we sought to compare the density of skin cell between transgenics and WT. To quantitate skin density, $\operatorname{Tg}(\mathrm{krt} 4 \text { :hcdc6 })^{\mathrm{cy} 24}$ and $\operatorname{Tg}(\mathrm{krt} 4$ : m.c-myc $\mathrm{T} 58 \mathrm{~A})$

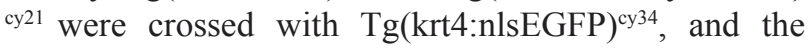
number of EGFP-positive skin cell nucleus in the trunk region was counted. The EGFP-positive cells enable us to calculate the skin cell number and density in a single cell resolution. We evaluated the skin cell density and morphology on embryos aged 3 day post-fertilization (dpf). Results showed that when cdc6 was overexpressed, there is no significant difference of skin cell density between WT $\left(1868 \pm 154 \mathrm{~mm}^{-2}\right)$ and $\operatorname{Tg}(\mathrm{krt} 4$ : krt4:hcdc6) cy24 $\left(1850 \pm 211 \mathrm{~mm}^{-2}\right)$ (Figs. 1A, B, and D). However, c-myc overexpression significantly increased the skin density $\left(3169 \pm 312 \mathrm{~mm}^{-2}\right)$ compared with WT $(1868 \pm 154$ $\mathrm{mm}^{-2}$ ) (Figs. 1A, C, and D). We next observed the skin morphology at histological level by using plastic section through the yolk sac region. We found that the skin of $\operatorname{Tg}(\mathrm{krt4} \text { : m.c-myc T58A) })^{\mathrm{cy} 21}(7.7 \pm 1.2 \mu \mathrm{m})$ is significantly thinner than WT $(11.6 \pm 1.5 \mu \mathrm{m})$ and $\operatorname{Tg}(\operatorname{krt} 4:$ krt4:hcde6) 
cy24 $(11.4 \pm 2.1 \mu \mathrm{m})$ (Figs. $1 \mathrm{E}$ to $\mathrm{G}$ and $\mathrm{H})$. These results clearly suggested that neither Cdc6 nor c-Myc overexpression only is sufficient to transform zebrafish skin although c-Myc induces skin hyperplasia.

\section{Co-overexpression of cdc6 and c-myc induces tumor-like transformation in zebrafish skin}

The failure to transform zebrafish skin in $\operatorname{Tg}(\text { krt4:hcdc6) })^{\text {cy } 24}$ and $\operatorname{Tg}\left(\right.$ krt4: m.c-mycT58A) ${ }^{\text {cy } 21}$ leads us to test whether co-overexpression of cdc6 and c-myc transforms zebrafish skin. The skin out-looking of progenies derived from either single or double transgenic line with nlsEGFP background was monitored by using the fish aged from 3 to $12 \mathrm{dpf}$. After examining skin morphology and histology, we were unable to detect any sign of skin oncogenic transformation in WT (Figs. 2A, E, I), cdc6 (Figs. 2B, F, J), or c-myc single transgenic lines at $12 \mathrm{dpf}$ (Figs. 2C, G, K). However, about 15\% progenies derived from cdc6 and c-myc double transgenic lines displayed tumor-like protruding structure on the skin surface (especially on the pectoral fin) from at only 8 dpf (Figs. 2D, H, L, S1B). In addition, all progenies with skin tumor phenotype were lethal and not survived longer than $15 \mathrm{dpf}$. Why is pectoral fin region so sensitive to skin tumor-like transformation? We hypothesized that the cell proliferative kinetics in the pectoral fin is much faster than the one in other tissue compartments. To prove this hypothesis, we performed BrdU incorporation (S phase marker) and pHistone3 staining (M phase marker) on cdc6/c-myc transgenics aged $8 \mathrm{dpf}$. Results support our speculation since the robust BrdU- or $\mathrm{pH} 3$-positive cells were observed in largely the domain of pectoral fin (Fig. S2A-D). In addition to the pectoral fin, we found that the anal and tail fins are a hot spot for active skin cell proliferation (Fig. S2E).

\section{Co-overexpression of cde6 and c-myc induces skin hyperplasia by increasing cell proliferation and attenuating apoptosis}

The intriguing oncogenic transformation phenotype in the skin of cdc6/c-myc double transgenics leads us to ask whether this is caused by acceleration of cell cycle and attenuation of cell death. High magnification view of the anal and tail fins showed that skin cells are aggregated
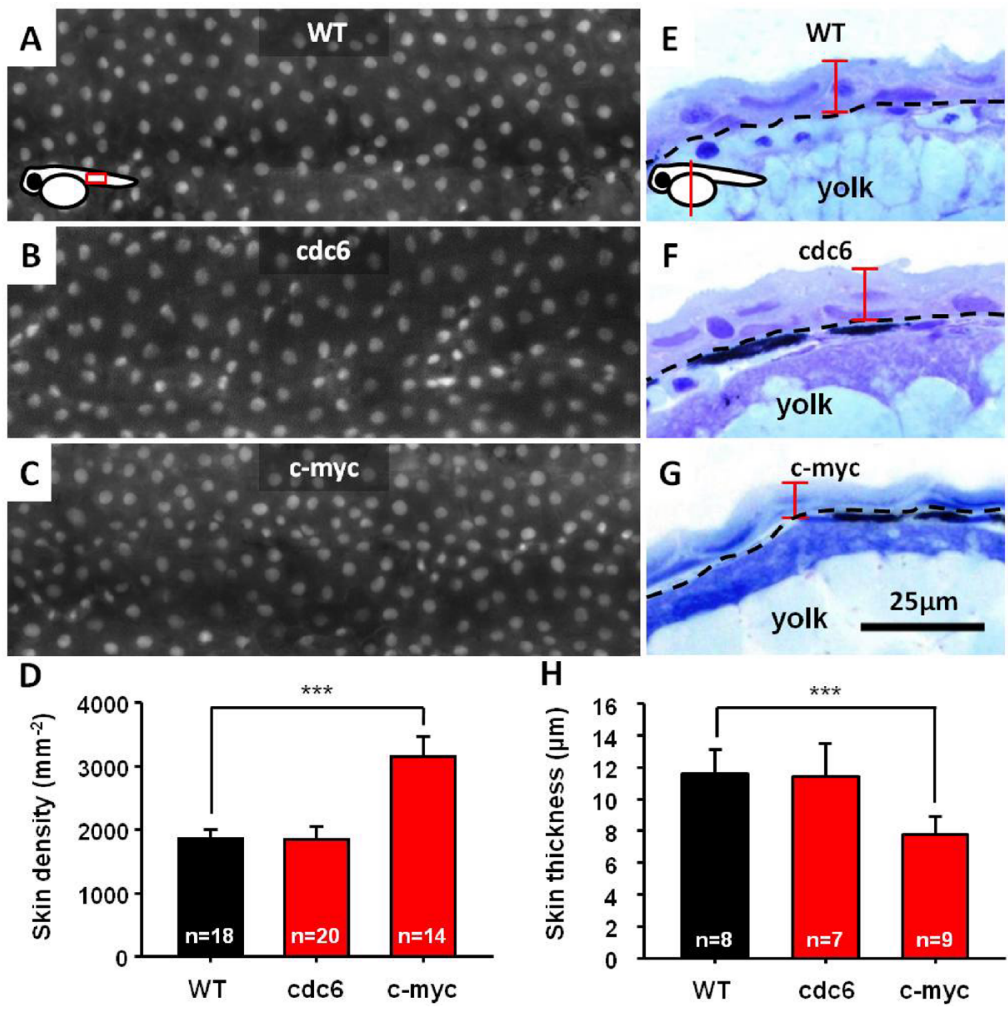

Figure 1: Overexpression of either cdc6 or c-myc is insufficient to transform zebrafish skin cells. (A-D) Comparison of the skin cell density between WT, cdc6- and c-myc-overexpressing fish embryos aged $3 \mathrm{dpf}$. To facilitate skin cell counting, WT, cdc6- and c-myc-overexpressing fish are cross with $\operatorname{Tg}(k r t 4: n l s E G F P){ }^{\text {cy } 34}$ and the skin cell density was compared for the embryos aged 3 dpf. (E-H) Comparison of the skin cell thickness between WT, cdc6- and c-myc-overexpressing fish embryos aged 3 dpf. WT, cdc6- and c-myc fish embryos were embedded in resin and conducted semi-thin section at $3 \mu \mathrm{m}$ intervals. The length of skin thickness calculated is highlighted by red color. Scale bar in $=25 \mu \mathrm{m}$. The significant difference of skin cell density (D) and thickness $(\mathrm{H})$ between embryos were conducted with t-test. 


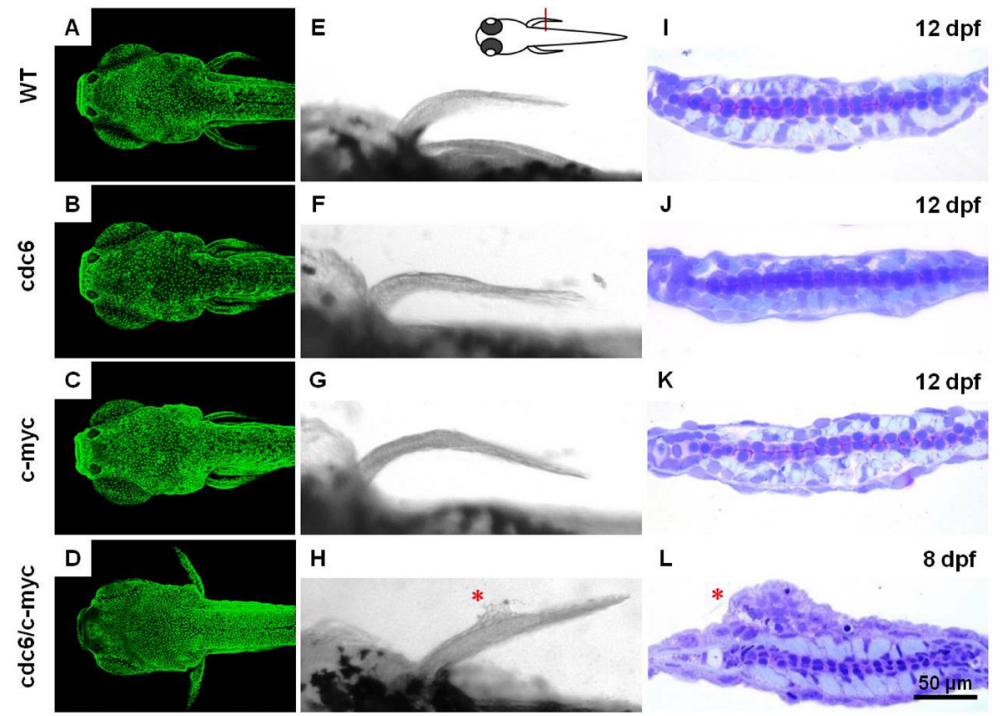

Figure 2: Co-overexpression of cdc6 and c-myc successfully transforms zebrafish skin cells. (A-D) Comparison of the skin cell density between different genetic backgrounds which carrying either (A) WT, (B) cdc6, (C) c-myc or (D)cdc6/c-myc transgenes. (E$\mathrm{H})$ Comparison of the gross morphology in the pectoral fin area in either (E) WT, (F) cdc6, (G) c-myc or (H) cdc6/c-myc transgenes. (I-L) Comparison of the histology in the pectoral fin area in either (I) WT, (J) cdc6, (K) c-myc or (L) cdc6/c-myc transgenes. The developmental stage that the embryos were sacrificed for histology assessment was labeled in the upper right corner. Embryos were crossed sectioned across the pectoral fins positions (highlighted by red line) and the sections were counterstained with H\&E. Scale bar $=100 \mu \mathrm{m}$ in the bright field images (left panel in all images). Scale bar $=50 \mu \mathrm{m}$ in the plastic sections (right panel in all images). In images from A to $\mathrm{H}$, anterior is positioned to the left.

A
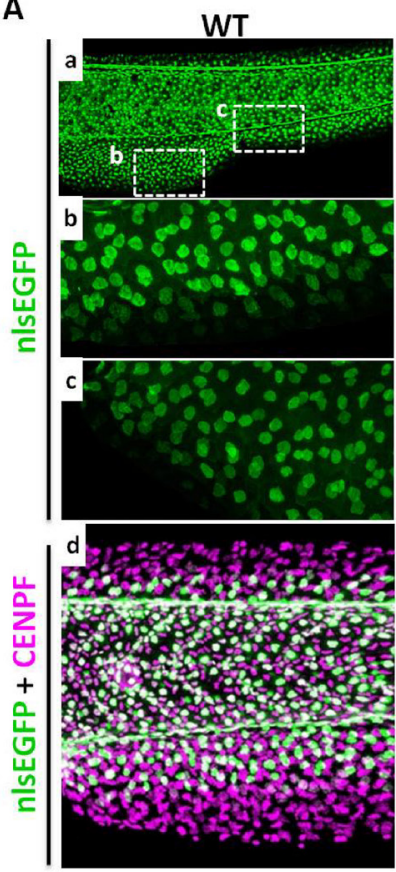

cdc6/c-myc
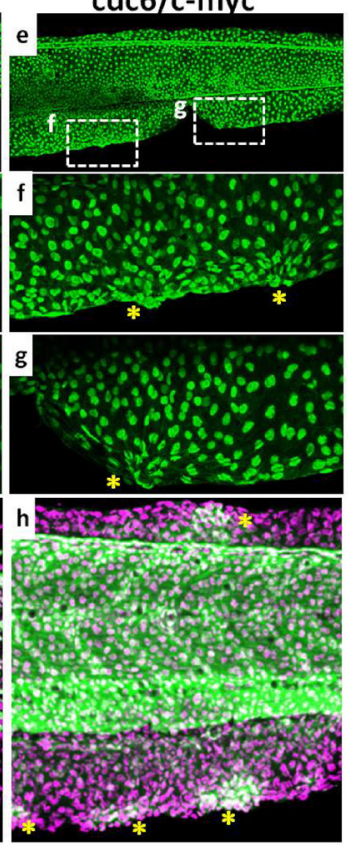

B

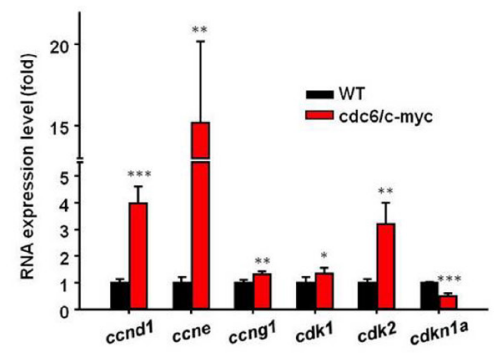

C

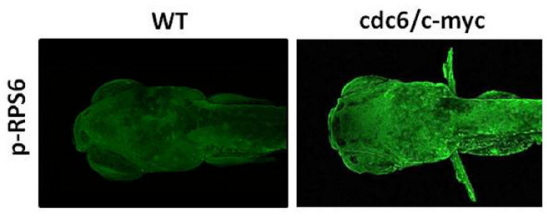

Figure 3: The skin cell morphology and cell proliferation activity in cdc6/c-myc transgenic fish. (A). The skin cell morphology in the anal and tail fin regions of WT (a-d) or cdc6/c-myc double transgenics (e-h) aged 8 dpf. To facilitate skin cell counting, WT, cdc6- and c-myc-overexpressing fish were crossed with $\operatorname{Tg}(k r t 4: n l s E G F P){ }^{\text {cy } 34}$ and their skin cell density were compared. The positions labeled by dotted line in the anal (f) and tail ( $\mathrm{g}$ ) fin of cdc6/c-myc transgenics were enlarged to show abnormal skin aggregation in cdc6/cmyc transgenics. The corresponding positions in WT were also shown (b and c). Total cell nuclei including skin cells were labeled by CENPF antibody (magenta color) ( $\mathrm{d}$ and $\mathrm{h}$ ) and skin cells were double labeled by CENPF and EGFP antibody (green color). Overexpression of cdc6/c-myc in skin cell can induce skin cell abnormal aggregation in dorsal and anal fin (stars in $\mathrm{f}, \mathrm{g}$, and h). (B) Comparison of the expression of cell cycle markers by real time RT-PCR. The cell cycle markers like ccnd1, ccne, and cdk2 were significantly upregulated while the cell cycle inhibitor cdkn1a was significantly downregulated. (C) Comparison of the protein expression of protein synthesis markers. The marker like p-RPS6 was greatly upregulated in cdc6/c-myc transgenics. Result is presented as a representative of at least three independent experiments. 
in the fin fold regions of cdc6/c-myc transgenics (e to $\mathrm{h}$ in Fig. 3A), while those in the WT fish are deposited in an organized order (a to d in Fig. 3A). By using antiGFP (krt4-positive cells) and anti-CENPF (cell nucleus labeling) double staining, krt4-positive cells were observed in the entire region of fin fold in cdc6/c-myc transgenic embryos (h in Fig. 3A), which might be caused by the migration of the cells from lateral epidermis to fin folds. However, in the wild-type embryos, we found that krt4-positive cells were only partially distributed in the fin fold region but never observed in the marginal region of the same area ( $d$ in Fig. 3A). To confirm active cell proliferation in skin cells from cdc6/c-myc transgenics at molecular level, we examined the gene expression of cell cycle-related markers. Results showed that cyclin D (ccnd1), cyclin E (ccne), cyclin G1 (ccng1), cyclindependent kinase 1 (cdk1), and CDK 2 (cdk2) were upregulated, while CDK inhibitor 1a (cdkn1a, p21/WAF1) was downregulated in the cdc6/c-myc transgenics (Fig. $3 \mathrm{~B})$. In addition, we noticed that the expression level of p-RPS6, a marker for protein translation, was highly upregulated in cdc6/c-myc lines, suggesting that protein synthesis is highly active in cde6/c-myc transgenic fish (Fig. 3C). We next further quantitated the skin cell proliferation in either WT or double transgenics aged $8 \mathrm{dpf}$ by BrdU incorporation using an image-based cytometry. Results showed that the cell proliferation activity in cdc6/ c-myc transgenics was much higher than the one in WT (Fig. 4A, B, G and S3). For example, a high magnification view in the ventral fin region showed that the BrdUpositive cells in cdc6/c-myc double transgenics were higher as 13 folds than the ones in WT (Fig. 4C and D). We concluded that co-overexpression of cdc6 and c-myc in zebrafish skin greatly accelerates cell proliferation.

To address apoptosis attenuation, we performed a whole-mount fluorescent TUNEL staining using 8dpf aged embryos of either WT or double transgenics. Results showed that physiological amounts of apoptotic cells were observed in the anal fin margins of WT (Fig. 4E) but not in cdc6/c-myc transgenics whose apoptotic cells were significantly abolished (Fig. 4F and H). Taken together, we concluded that co-overexpression of cdc6 and c-myc in
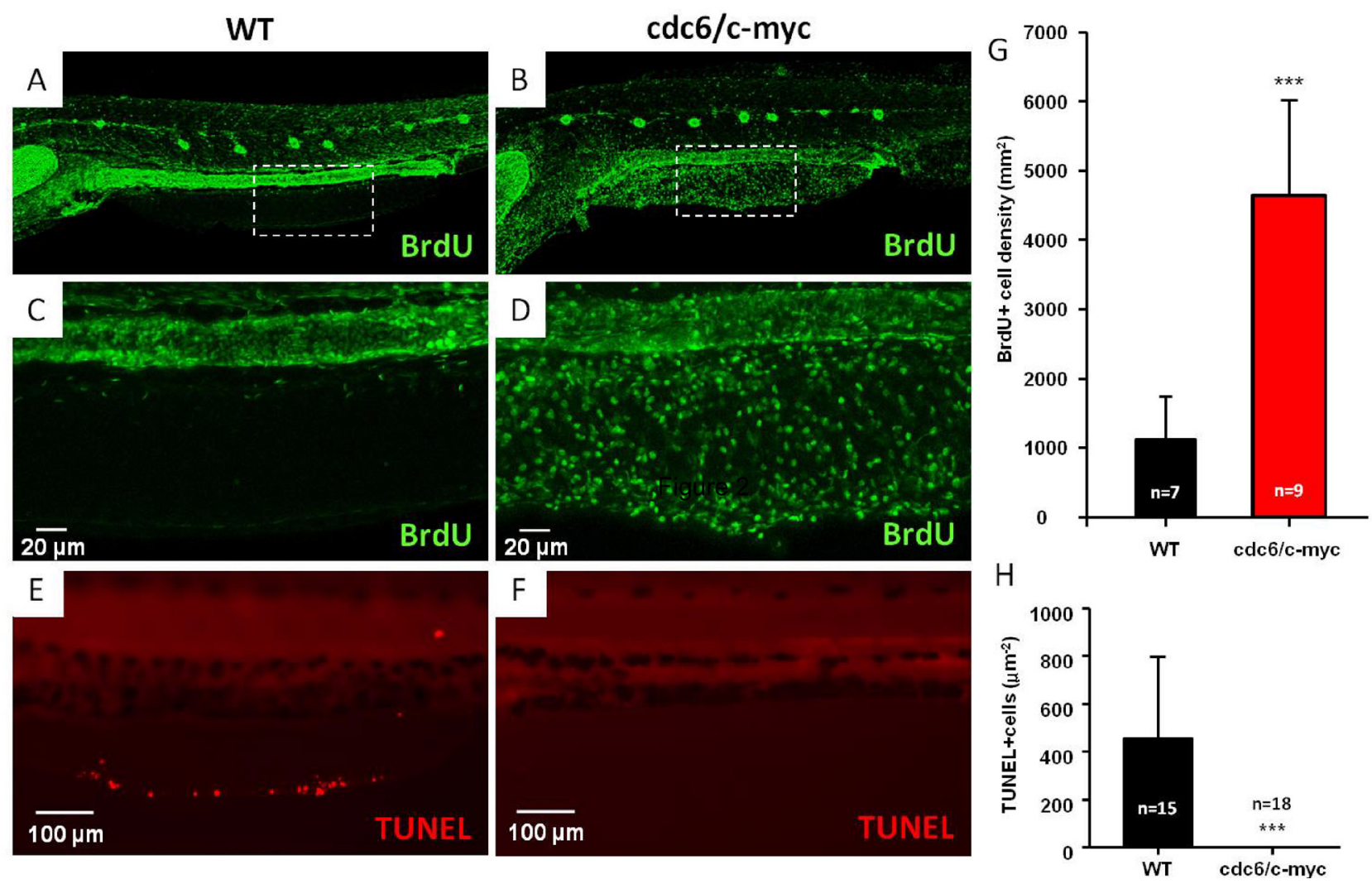

Figure 4: Comparison of cell proliferation and apoptosis in WT and cdc6/c-myc transgenic zebrafish. The BrdU incorporation experiment was conducted to compare the cell proliferation index between WT (A and C) and cdc6/c-myc double transgenic fish (B and D). Embryos aged $7 \mathrm{dpf}$ were incubated with $10 \mathrm{mM} \mathrm{BrdU}$ for $24 \mathrm{hr}$ and later washed with fish water and finally fixed with PFA at $8 \mathrm{dpf}$ and proceed for anti-BrdU antibody staining. The images highlighted by dotted lines in the ventral fin (A and B) were magnified in $\mathrm{C}$ and $\mathrm{D}$, which show the cell patterns with positive BrdU-labeling. The area of BrdU + cells was quantified by image $\mathrm{J}$ software and statistically presented in $(\mathrm{G})$ for comparison. Scale bar $=20 \mu \mathrm{m}$ in $\mathrm{C}$ and $\mathrm{D}$. The TUNEL assay was conducted to compare the apoptotic index between WT (E) and cdc6/c-myc double transgenic fish (F). The area of TUNEL+ cells was quantified by image J software and statistically presented in $(\mathrm{H})$ for comparison. Scale bar $=100 \mu \mathrm{m}$ in E and F. In all images, anterior is positioned to the left. The significance was compared with student T-test. 
zebrafish skin accelerates cell proliferation and attenuates apoptotic cell death, finally giving an increase in the hyperplastic growth of skin cells.

\section{Detection of nuclear aberration and genomic instability in cdc6/c-myc double transgenics}

Overexpression of Cdc6 and c-Myc leads to polyploidy and genomic instability in a variety of cancers $[5,6,9,25]$. To examine the transformation phenotype in zebrafish skin, we performed plastic section through the tumor-like structure in the anal fin area of cdc6/c-myc transgenics. Compared to WT, the nucleus morphology of cde6/c-myc fish displays severe abnormality (Fig. 5A and B). By measuring the nuclear size of the skin cells, nuclear pleomorphism was detected in cdc6/c-myc transgenics. In WT, the nuclear size displays normal distribution pattern with an average nuclear size at $31 \pm 3 \mu \mathrm{m}^{2}$. However, only in cdc6/c-myc transgenics, some macronucleoli (nuclear size larger than $100 \mu \mathrm{m}^{2}$ ) and micronucleus (nuclear size smaller than $10 \mu \mathrm{m}^{2}$ ) were detected (Fig. 5C and D).

The nuclear atypia phenotype detected in cdc6/ c-myc transgenic embryos strongly suggests it may accompany with genomic instability problem. To validate this hypothesis, we performed image-based cytometry and

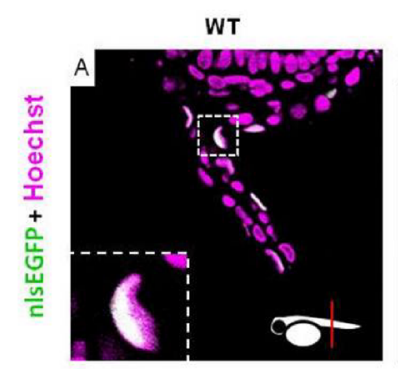

E

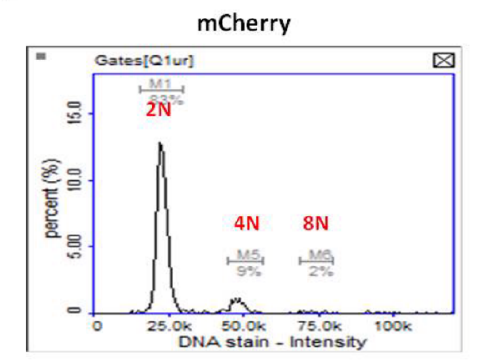

$\mathbf{F}$

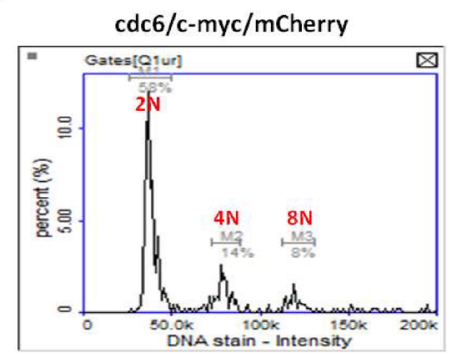

H

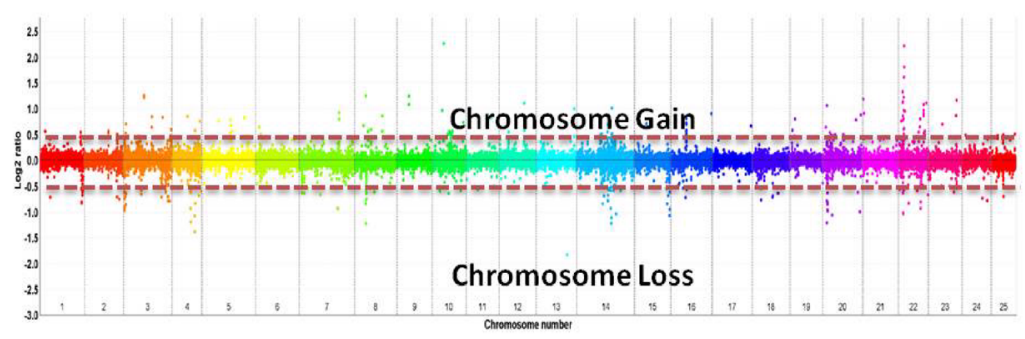

D
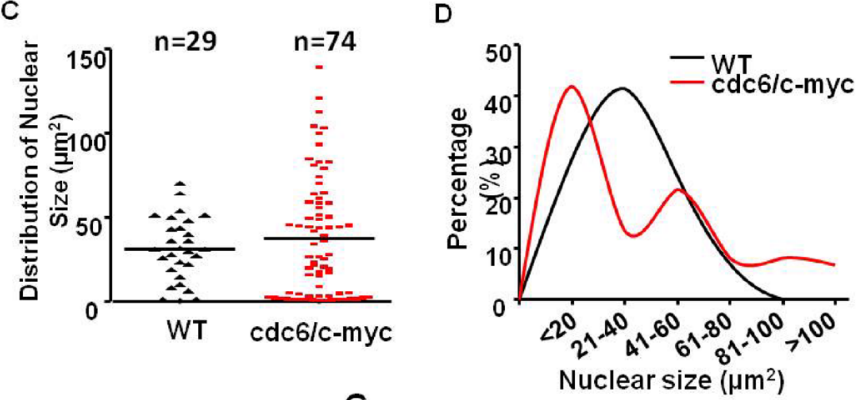

G

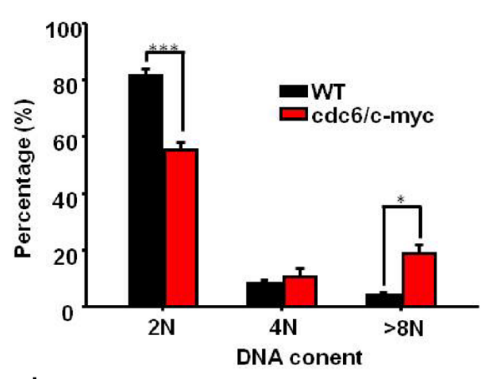

I

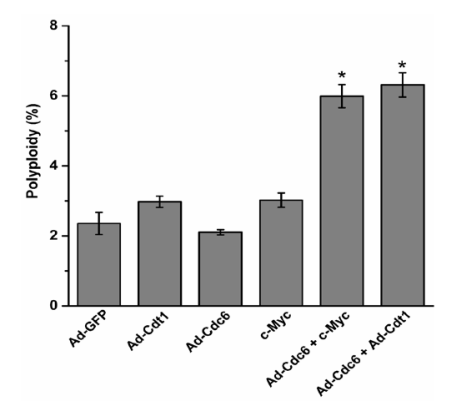

Figure 5: cde6/c-myc co-overexpression induces polyploidy and chromosomal instability in transgenic zebrafish and human cell. Detection of skin morphology in the pectoral fin region of WT (A) and cdc6/c-myc (B) transgenic zebrafish embryos aged at $8 \mathrm{dpf}$. The skin cells were visualized by nlsEGFP siganls (green color) and the cell nuclei were counterstained by Hoechst staining (pseudocolor in magenta). The normal skin cell nucleus was shown in flat and distributed in the outermost layer (A). The micronuclei and nucleus aberration were observed in the skin of ventral fin region of cdc6/c-myc double transgenics (B). The micronuclei (highlighted by arrows) were magnified and highlighted in the left lower corner. (C) The distribution of nuclear size in WT and cdc6/c-myc double transgenics. (D) The percentage of nuclear size in WT and cdc6/c-myc double transgenics. Scale bar $=20 \mu \mathrm{m}$ in A and B. Comparison of the DNA content in skin cells derived from either WT (E) or cdc6/c-myc double transgenic fish (F) aged at 8 dpf by using image based cytometry (N=10). (G) Statistic analysis of different DNA content in skin cells derived from WT and cdc6/c-myc double transgenic fish. Result showed that the $2 \mathrm{~N}$ content was decreased by $26.34 \%$ and the $>8 \mathrm{~N}$ content increased by $14.6 \%$. (H) Evaluations of the chromosomal stability of WT and cdc6/c-myc double transgenic fishes aged $8 \mathrm{dpf}$ by using array CGH. (I). Co-overexpression of Cdc6 and c-Myc in human cells induces polyploidy. U2OS cells were infected with Ad-Cdc6 $\left(1.2 \times 10^{7} \mathrm{pfu} / \mathrm{ml}\right)$, Ad-Cdt1 $\left(1.2 \times 10^{7} \mathrm{pfu} / \mathrm{ml}\right)$ and $/$ or Ad-Vec $\left(\right.$ Ad-GFP, $1.2 \times 10^{7}$ $\mathrm{pfu} / \mathrm{ml}$ ) and/or transfected with c-Myc expression plasmid. The cell cycle profile was obtained by FACS analysis $48 \mathrm{~h}$ after infection or transfection. The percentage of cells containing more than $4 \mathrm{~N}$ of DNA content is calculated and presented as polyploidy. 
array-based comparative genomic hybridization (aCGH) to detect the DNA content and chromosome stability of the transformed skin, respectively. For DNA content analysis, cdc6/c-myc transgenic embryo cells with H2AFZmCherry background aged 8dpf were gently dissociated, stained with Hoechst 33342 nuclear dye, and then subjected to image-based cytometry. For WT, both the skin and non-skin cell fractions display normal DNA content histogram (Fig. 5E and G). However, in cdc6/c-myc transgenic embryos, skin cells display typical polyploidy that is characterized with abnormal accumulation of $8 \mathrm{~N}$ fractions (Fig. 5F and $\mathrm{G}$ ). To further confirm genome instability in the zebrafish skin cells, we isolated genomic DNA from WT and cdc6/c-myc transgenic embryos aged 8 dpf, labeling genomic DNA with Cy3 and Cy5 dyes. The genomic DNA then was subjected to perform aCGH analysis with zebrafish $385 \mathrm{~K}$ CGH microarray. Results showed that cde6/c-myc transgenic embryos have abnormal subchromosomal amplification in Chromosome 10 and 22 (Fig. 5H).

To confirm the phenomenon of polyploidy observed in the zebrafish model, we tried to co-overexpress Cdc6 and c-Myc in human cells. After infecting cells with recombinant adenoviruses (Ad-Cdc6 or Ad-Cdt1), rereplication was monitored by accumulation of cells containing more than $4 \mathrm{~N}$ DNA content as described [26], which presents a form of replication stress, fuelling polyploidy and genomic instability. Co-overexpression of Cdc6 with c-Myc or Cdt1 significantly induced DNA rereplication (Fig. 5I). These results support the idea that co-overexpression of cdc6 and c-myc induces polyploidy and genomic instability, hallmarks of malignant tumorigenesis.

\section{Detection of ectopic skin cells and EMT in cde6/c- myc double transgenics}

To provide more evidences to support the skin transformation phenotype, we performed plastic section through the tumor-like tissue in the pectoral fin of cdc6/cmyc transgenics. In the section crossing pectoral fin of WT with nlsEGFP-positive background, we found the EGFPpositive skin nucleus is flat and restrictedly located on the superficial layer (a in Fig. 6A). Intriguingly, some ectopic EGFP-positive skin cells were detected in the internal location of the pectoral fin of cdc6/c-myc transgenics ( $d$ in Fig. 6A, highlighted by yellow stars).

In addition, severe disorganization of the muscle and cartilage layers was also noticed in cdc6/c-myc
A

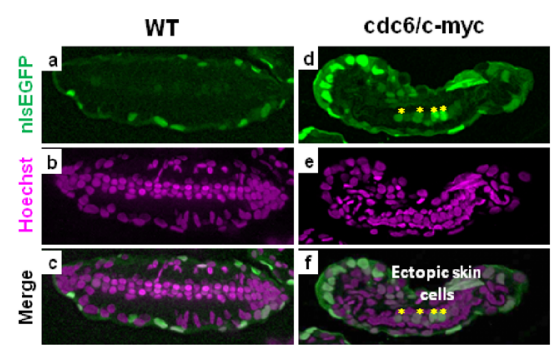

D

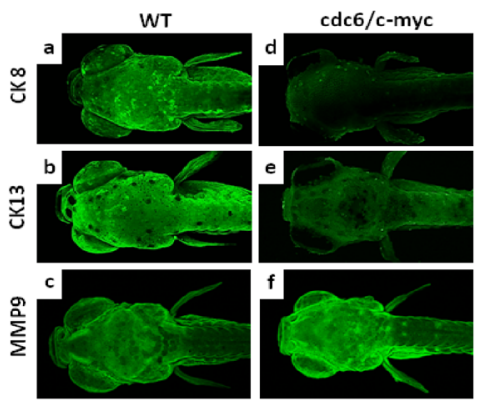

B

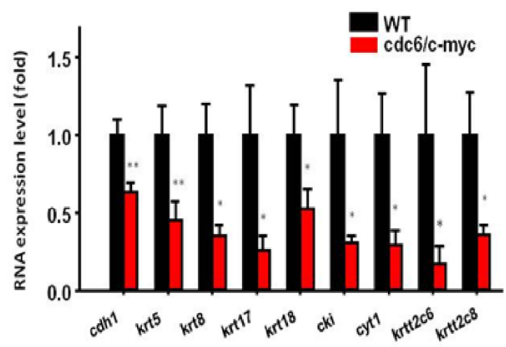

E

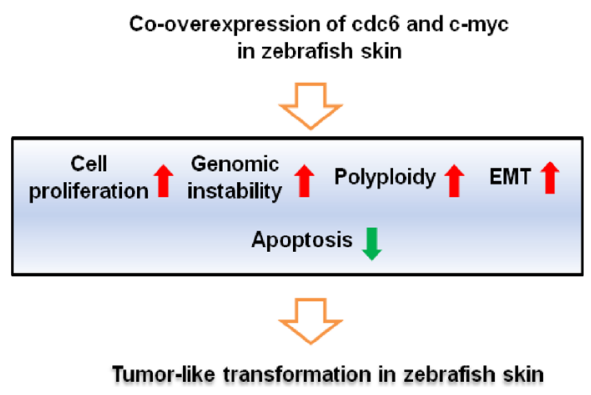

Figure 6: Deregulation of EMT markers and detection of polyploidy in the pectoral fin of cdc6/c-myc transgenic zebrafish and in human cancer cells. (A). The skin cells of WT (a-c) and cdc6/c-myc double transgenics (d-f) aged 8 dpf were labeled by nlsEGFP (green color) and co-staining with Hoechst 33342 nuclear dye in the pectoral fin sections. In normal fish, skin cells were restrictedly located in the outmost layer $(\mathrm{a}, \mathrm{c})$. However, some ectopic nlsEGFP+ cells (indicted by asterisks) were mixed with the inner cartilage tissues (d, f). (B) Real-time PCR results showed the epidermal markers of keratin gene family were significantly downregulated in cdc6/c-myc double transgenic embryos aged 8 dpf. (C) Real-time PCR results showed the mesenchymal markers were deregulated in cdc6/ c-myc double transgenics. MMP9 was highly expressed in cdc6/c-myc double transgenics. (D) Comparison of the EMT marker expression at protein levels. Epidermal markers like CK8 (a, d) and CK13 (b, e) were greatly downregulated while mechenchymal marker like MMP9 (c, f) was highly upregulated in cdc6/c-myc double transgenics. Result is presented as a representative of at least three independent experiments. (E). Summary of the phenotype detected in cdc6/c-myc double transgenic fish. EMT, epithelial to mesenchymal transition. 
transgenics (e in Fig. 6A). In many types of cancer, EMT transformation featured by down-regulation of epithelial markers and up-regulation of mesenchymal makers is frequently detectable and strongly associated with cancer malignancy [27]. The disorganization of skin cell distribution and the appearance of ectopic skin cells may suggest that the transformed skin cells are undergoing EMT. To clarify this speculation, we examined the mRNA expression profile of key genes related to EMT by realtime RT-PCR on embryos aged $8 \mathrm{dpf}$. Results showed that most epithelial markers, E-cadherin (cdhl) and type I keratin (cki, cyt1, krt17, krt18) and type II keratin (krt5, $k r t 8, k r t t 2 c 6$, and $k r t t 2 c 8)$, were downregulated in cdc6/ c-myc lines (Fig. 6B). In contrast, mesenchymal gene transcripts of mmp9, snailla, snaillb, snail2, twist1a, and twist $1 b$ were significantly upregulated in cdc6/c-myc lines (Fig. 6C). We further confirmed this EMT phenomenon by immunofluorescent staining. Agreed with mRNA expression, the relative expression level of epithelial markers of cytokeratin 8 (CK8) and cytokeratin 13 (CK13) was robust expressed in WT while they were barely detected in cdc6/c-myc lines (a and b in Fig. 6D). Again, the mesenchymal maker MMP9 was highly expressed in cdc6/c-myc lines but not in WT (c and f in Fig. 6D). These results strongly suggest that co-overexpression of cdc6 and c-myc in zebrafish skin induces EMT, a hallmark of malignant invasion and metastasis in human cancer.

\section{DISCUSSION}

In this work, we established a novel non-melanoma skin cancer model in zebrafish to study the oncogenic mechanisms of Cdc6 and c-Myc by co-overexpression of both in the outmost skin layer, rendering the practicability in a good cancer model and anti-cancer drug screening. Our studies suggest that co-overexpression of Cdc6 and c-Myc in zebrafish skin causes skin transformation through accelerated cell proliferation, apoptosis attenuation, genomic instability, and EMT (Fig. 6E).

In this study, we successfully created an early onset and a non-melanoma skin cancer model by specifically cooverexpressing two oncogenic genes that cause unbalance of cell cycle progression. Skin is the outmost tissue and provides a good model to evaluate the oncogenic transformation in living animal in a non-invasive manner. In addition, the outmost nature also makes it is easy to evaluate the biological effect of anti-cancer compound against cancer. In zebrafish, although many studies were attempting to induce skin cancer formation, no successful case was found in any skin cancer models except only in melanoma model [28]. Since we were failed to transform zebrafish skin cells by overexpression of single oncogene, we then tried to co-express two oncogenes to overcome the refractory threshold in zebrafish skin. Using cooverexpression of cdc6 and c-myc, we can induce tumor-like formation on the skin as early as from $8 \mathrm{dpf}$ onwards even in the WT fish with a normal p53 expression background. This cdc6/c-myc transgenic line will be an excellent experimental animal model and an anti-cancer drug screening platform for identifying the potent c-Myc or Cde6 inhibitor.

Our findings suggested that c-Myc only is able to induce skin hyperplasia but not sufficient to transform zebrafish skin. c-Myc is a transcription factor that directly modulates transcription of a large number of genes involved in cell cycle regulation, protein synthesis, metabolism, DDR, and apoptosis [29, 30]. The profound functions of c-Myc in tumorigenesis have been mainly attributed to its ability to coordinate transcription. In addition, deregulated expression of c-Myc leads to the generation of double strand breaks (DSBs), DDR and thus promotes genome instability [31]. Therefore, we suggest the reason of the failure of skin transformation induced by c-Myc overexpression only may be that cell transformation induced by oncogenic activation is restrained by cell cycle arrest, cellular senescence, and apoptosis, just like ras and $\operatorname{mos}[11,12]$.

Next we found that co-overexpression of cdc6 and c-myc induces tumor-like transformation in zebrafish skin. The mechanisms could be increased cell proliferation, apoptosis attenuation, and the effect of genomic instability. Several explanations have been proposed to elucidate the phenomenon of genomic instability triggered by c-Myc overexpression, including inducing DNA replication stress [9, 32, 33], inhibiting DNA repair activities [34], and increased generation of reactive oxygen species (ROS) [25]. In addition, a recent study has identified a different mechanism for c-Myc in the direct stimulation of DNA replication, independent of its transcriptional targets. $\mathrm{c}-\mathrm{Myc}$ is able to bind to components of the pre-RC, including $\mathrm{Cdc} 6$, and bind to known mammalian replication origins [9]. The genetic interaction between c-Myc and Cdc6 observed in the double transgenic zebrafish could be elucidated as the following. First, c-Myc and Cdc6 overexpression may enhance replication stress through both transcription-dependent and -independent function of c-Myc. Our results support the oncogenic potential of overexpressed c-Myc and Cdc6 from the earliest stages of skin transformation, which may be achieved by active replication stress. Initially, the stress activates the antitumor barriers of senescence or apoptosis (Myc or Cdc6 overexpression only), but the barriers eventually are bypassed by the continuous co-overexpression of c-Myc and Cdc6, which induces genomic instability favoring clonal expansion of cells with more aggressive properties. Second, Cdc6 overexpression may regulate the switch between the transcription-dependent and -independent function of c-Myc, as a study [13] showed that $\mathrm{Cdc} 6$ overexpression interferes with the interaction between c-Myc and Max, which inhibits E-boxdependent transcription activity of c-Myc. Third, Cdc6 overexpression may increase replication efficiency and 
gene expression of $c-m y c$ gene because a recent study showed that Cde6 is increased its binding to the replication origins of $c-m y c$, and the binding augments the origin activity [35].

Intriguingly, we observed that co-overexpression of cdc6 and c-myc induces EMT in zebrafish skin. Consistently, in cdc6/c-myc transgenic lines, the gene expression of epithelial markers, E-cadherin and cytokeratin, were downregulated; the gene expression of mesenchymal markers, MMP9, Snail, Twist1 were significantly upregulated. This result is supported by the finding that overexpressed $\mathrm{Cdc} 6$ binds directly to the E-boxes of the $C d h 1$ promoter and represses its transcription [14].

\section{MATERIALS AND METHODS}

\section{Fish lines and plasmid construction}

The detailed procedure for fish lines and plasmid construction used in this study have been described in previous publication [36] and in Supplementary Data.

\section{Image Acquisition, Skin Cell Quantification and Statistics}

Representative fluorescent images were acquired using an upright microscope (BX51, Olympus) equipped with a digital camera (DP72, Olympus) or a dissecting microscope (SMZ1500, Nikon) equipped with a cool CCD (Evolution VF). For quantifying the relative density of skin cells, the original images captured at the trunk position were processed using Photoshop CS3 software to select a region of interest (ROI) at $150 \mu \mathrm{m}$ x $450 \mathrm{~m}$ dimensions. The total cell number in this ROI was calculated using ImageJ software and statistically compared using t-test or one-way ANOVA.

\section{Whole-Mount Immunostaining and Histology}

Zebrafish embryos aged specific developmental stages were fixed in $4 \%$ paraformaldehyde/PBS for 12 $\mathrm{h}$ at $4^{\circ} \mathrm{C}$. After extensive washing in PBST, embryos were transferred to $100 \%$ methanol at $-20^{\circ} \mathrm{C}$ for $2 \mathrm{~h}$ and subsequently subjected to rehydration with PBST. After blocking with $3 \% \mathrm{BSA} / \mathrm{PBST}$ at room temperature for 60 min, embryos were incubated at $4^{\circ} \mathrm{C}$ overnight with 1:200 diluted primary antibodies as follows: rabbit anti-pH3 (sc8656-R, Santa Cruz), rabbit anti-human phosphorylated ribosomal Protein S6 (Ser235/236) (GTX113542, GeneTex), rabbit anti-human CK8 (GTX110311, Genetex), rabbit anti-human CK13 (GTX109883, Genetex), rabbit anti-human MMP9 (GTX100626,
GeneTex), and rabbit anti-human CENPF (GTX100212, Genetex). After extensive washing in PBST for $10 \mathrm{~min}$, embryos were incubated with 1:500 diluted Alexa Fluor 488 or 568 -conjugated secondary antibodies (Invitrogen) for fluorescent detection of immunoreactive signals. For histology, some stained embryos were further infiltrated and embedded in Technovit 7100 resin (Heraeus Kulzer). Samples were sectioned at $3 \mu \mathrm{m}$ intervals and counterstain with either Hoechst 33342 (Invitrogen) or SYTOX orange (Invitrogen) to visualize the nuclear position.

\section{Real-time quantitative PCR}

Ten zebrafish larvae aged at $8 \mathrm{dpf}$ from wild-type or double transgenic lines were collected and homogenized in RNAzol RT (RN190, MRC, Inc) with Bullet Blender (Next Advance, Inc) tissue lyser to isolate total RNA according to the manufacturer's instructions. Total RNA concentration was determined by spectrophotometry, and the RNA quality was checked by electrophoresis in RNA denatured gels. For RT-PCR, $1 \mu \mathrm{g}$ of total RNA was reverse-transcribed with RevertAid first cDNA synthesis kit (K1622, Fermentas) and then PCR was performed with SYBR green dye according to the manufacturer's instructions. The PCR condition: $95^{\circ} \mathrm{C}(15 \mathrm{~s}), 60^{\circ} \mathrm{C}(1$ $\mathrm{min}), 72^{\circ} \mathrm{C}(1 \mathrm{~min})$ for 40 cycles, and all reactions were started at $95^{\circ} \mathrm{C}$ for $5 \mathrm{~min}$ and terminated at $72^{\circ} \mathrm{C}$ for $5 \mathrm{~min}$. The primer sequences used to perform RT-qPCR and the PCR amplicon size are listed in Table S1.

\section{BrdU Incorporation}

Zebrafish embryos aged 7 dpf were systematically incubated in $10 \mathrm{mM} \mathrm{BrdU}$ (B5002, Sigma)/5\% DMSO/ PTU solution for $24 \mathrm{hr}$. After fixation, embryos aged 8 dpf were depurated with $2 \mathrm{~N} \mathrm{HCl}$ for $60 \mathrm{~min}$ at room temperature and immersed in $0.1 \mathrm{M}$ borate buffer for 10 min to enhance the sensitivity for detection of the BrdUpositive cells in skin. Later, embryos were subjected to staining with mouse anti-BrdU antibody $(1: 100, \mathrm{G} 3 \mathrm{G} 4$, DSHB) and Alexa Fluor 488-conjugated goat anti-mouse secondary antibody (Invitrogen).

\section{TUNEL assay}

For TUNEL assay, embryos aged 18 hour postfertilization (hpf) were pre-treated with PTU and then processed to PFA fixation at $48 \mathrm{hpf}$. Following washing in PBST for $10 \mathrm{~min}$ they were then stored in $100 \%$ methanol at $-20^{\circ} \mathrm{C}$ for over $2 \mathrm{~h}$. Embryos were incubated with $3 \% \mathrm{H}_{2} \mathrm{O}_{2} / \mathrm{MeOH}$ for $10 \mathrm{~min}$ at room temperature. Subsequently, embryos were rinsed two times with PBS and incubated with labeling solution, $10 \mu \mathrm{L}$ of enzyme solution plus $90 \mu \mathrm{L}$ of label solution at $37^{\circ} \mathrm{C}$ for $2 \mathrm{~h}$ 
following the kit instructions (Roche Applied Sciences). Embryos were then washed three times in PBS, for 5 min each time, at room temperature. Subsequently, embryos were incubated with $100 \mu \mathrm{L}$ of converter POD at $37^{\circ} \mathrm{C}$ for $30 \mathrm{~min}$. Embryos were rinsed three times in PBS and incubated with DAB solution (SK-4100, Vector Laboratories) for $5 \mathrm{~min}$.

\section{Image-based cytometry}

To facilitate skin cell counting, WT or cdc6+c-myc double transgenics were crossed with $\mathrm{Tg}(\mathrm{krt} 4: \mathrm{mCherry})$ cy9. To enhance the detection sensitivity and reduce the total amount of embryos used to analysis the cell cycle and DNA content, we adapted an imaged-based cytometry approach by using NucleoCounter NC-3000 FlexiCyte machine (Chemometec, Denmark). Initially, ten embryos aged $8 \mathrm{dpf}$ were slightly fixed in 4\%PFA for $20 \mathrm{~min}$ at room temperature and then dissociated with $250 \mathrm{uL}$ lysis buffer at $37^{\circ} \mathrm{C}$ for $10 \mathrm{~min}$. The cell lysate was later incubated with $250 \mathrm{uL}$ stabilization buffer to neutralize the $\mathrm{pH}$ value. After this cell dissociation process, the mCherry fluorescence is gone and we performed immunofluorescent staining with rabbit anti-mCherry primary antibody (1:200, GTX5788, Genetex) and goat anti-rabbit IgGAlexa Flour 568-conjugated secondary antibody (1:500, Invitrogen) to recover the skin fluorescence. Finally, all dissociated cells were counter-stained with Hoechst 33342 (H1399, Invitrogen) at $5 \mathrm{ppm}$ concentration at $37 \mathrm{C}$ for $1 \mathrm{hr}$ to illuminate cell nucleus and then loaded onto chamber slide (NC-Slide A2) for taking images. The DNA content and cell cycle were analyzed Nucleoview NC-3000 software according to manufactory instruction (Chemometec, Denmark).

\section{Array CGH and Data Processing}

Genomic DNA was isolated from WT and cdc6/ c-myc transgenic embryos aged $8 \mathrm{dpf}$ according to standard protocol [37]. Genomic DNA samples were then subjected to perform quality check by spectrophotometry (NanoDrop ND-1000, Thermo Fisher. Scientific, Inc.) and DNA electrophoresis. High quality genomic DNA which pass the quality check were labeled with $\mathrm{Cy} 3$ and Cy5 fluorescent dyes by using NimbleGen DualColor DNA Labeling Kit. Reference DNA was labeled with Cy5-random nonamers while test DNA was labeled with Cy3-random nonamers through klenow reaction. Hybridization assays were performed according to the manufacturers' instructions by using NimbleGen zebrafish CGH $385 \mathrm{~K}$ Whole Genome-Tiling array. The zebrafish whole genome tiling array contains 65,536 probes which designed according to the Zv6 assembled genome. Hybridization reaction between probe and array was conducted with NimbleGen Hybridization
Kit and NimbleGen Hybridization system to provide an active mixing action for $48 \mathrm{hrs}$ and constant incubation temperature at $42^{\circ} \mathrm{C}$. The hybridization result was scanned with NimbleGen MS200 microarray scanner automatically taking optimized signal in $2 \mu \mathrm{m}$ pixel resolution and the relative fluorescent intensity was normalized, converted to $\log 2$ scale by NimbleScan software. The segmented $\mathrm{CGH}$ data were generated by $\mathrm{CGH}-\mathrm{segMNT}$ analysis of NimbleScan software.

\section{Adenovirus construction and infection}

Adenoviruses encoding GFP (Ad-Vec), human Cdc6 (Ad-Cdc6) and Cdt1 (Ad-Cdt1) were generated and purified by using the AdEasy system [38] and as described previously [39]. Large-scale purification of adenovirus from 293 cells was performed by $\mathrm{CsCl}$ density gradient centrifugation. The concentration of purified virus was measured $\mathrm{OD}_{260}$ using the equation $1 \mathrm{OD}_{260} \approx 10^{12} \mathrm{pfu}[40]$.

The detailed procedures for cell culture and microinjection and identification of transgenic Zebrafish used in this study are described in Supplementary Data.

\section{Competing interests statement}

The authors have no conflicts of interest to declare.

\section{ACKNOWLEDGMENTS}

We thank Dr. Igea D’Agnano for kindly providing pcDNA3-c-Myc plasmid.

\section{Funding}

This work was supported by grants from National Health Research Institutes, Taiwan (102A1-CA-PP-08 and 103A1-CA-PP-07 to A. Y.-L. Lee), Ministry of Science and Technology 98-2311-B-400-003-MY3, 99-2815-C-400-001-B, and 102-2320-B-400-014 (to A. Y.-L. Lee); 102-2311-B-033-001-MY3, 103-2622-B-033001-CC2, and 103-2622-B-033-002-CC2 (to C.D. Hsiao), Department of Health, Taiwan DOH99-TD-C-111-004 (to A. Y.-L. Lee), and Mackay Memorial Hospital MMH-E-101-13, MMH-E-102-02, and MMH-CY-10204 (to D.S. Lin).

\section{REFERENCES}

1. Bell SP and Dutta A. DNA replication in eukaryotic cells. Annu Rev Biochem. 2002; 71:333-374.

2. Kelly TJ and Brown GW. Regulation of chromosome replication. Annu Rev Biochem. 2000; 69:829-880.

3. Takai H, Xie Y, de Lange $\mathrm{T}$ and Pavletich NP. Tel2 structure and function in the Hsp90-dependent maturation 
of mTOR and ATR complexes. Genes \& development. 2010; 24(18):2019-2030.

4. Hanahan D and Weinberg RA. Hallmarks of cancer: the next generation. Cell. 2011; 144(5):646-674.

5. Liontos M, Koutsami M, Sideridou M, Evangelou K, Kletsas D, Levy B, Kotsinas A, Nahum O, Zoumpourlis V, Kouloukoussa M, Lygerou Z, Taraviras S, Kittas C, Bartkova J, Papavassiliou AG, Bartek J, et al. Deregulated overexpression of hCdt1 and hCdc6 promotes malignant behavior. Cancer Res. 2007; 67(22):10899-10909.

6. Pinyol M, Salaverria I, Bea S, Fernandez V, Colomo L, Campo E and Jares P. Unbalanced expression of licensing DNA replication factors occurs in a subset of mantle cell lymphomas with genomic instability. Int J Cancer. 2006; 119(12):2768-2774.

7. Gonzalez S, Klatt P, Delgado S, Conde E, Lopez-Rios F, Sanchez-Cespedes M, Mendez J, Antequera F and Serrano M. Oncogenic activity of Cdc6 through repression of the INK4/ARF locus. Nature. 2006; 440(7084):702-706.

8. Borlado LR and Mendez J. CDC6: from DNA replication to cell cycle checkpoints and oncogenesis. Carcinogenesis. 2008; 29(2):237-243.

9. Dominguez-Sola D, Ying CY, Grandori C, Ruggiero L, Chen B, Li M, Galloway DA, Gu W, Gautier J and DallaFavera R. Non-transcriptional control of DNA replication by c-Myc. Nature. 2007; 448(7152):445-451.

10. Tatsumi Y, Sugimoto N, Yugawa T, Narisawa-Saito M, Kiyono $\mathrm{T}$ and Fujita M. Deregulation of Cdt1 induces chromosomal damage without rereplication and leads to chromosomal instability. J Cell Sci. 2006; 119(Pt 15):31283140 .

11. Bartkova J, Rezaei N, Liontos M, Karakaidos P, Kletsas D, Issaeva N, Vassiliou LV, Kolettas E, Niforou K, Zoumpourlis VC, Takaoka M, Nakagawa H, Tort F, Fugger $\mathrm{K}$, Johansson F, Sehested M, et al. Oncogene-induced senescence is part of the tumorigenesis barrier imposed by DNA damage checkpoints. Nature. 2006; 444(7119):633637.

12. Di Micco R, Fumagalli M, Cicalese A, Piccinin S, Gasparini P, Luise C, Schurra C, Garre M, Nuciforo PG, Bensimon A, Maestro R, Pelicci PG and d'Adda di Fagagna F. Oncogeneinduced senescence is a DNA damage response triggered by DNA hyper-replication. Nature. 2006; 444(7119):638-642.

13. Takayama M, Taira T, Iguchi-Ariga SM and Ariga H. CDC6 interacts with c-Myc to inhibit E-box-dependent transcription by abrogating c-Myc/Max complex. FEBS Lett. 2000; 477(1-2):43-48.

14. Sideridou M, Zakopoulou R, Evangelou K, Liontos M, Kotsinas A, Rampakakis E, Gagos S, Kahata K, Grabusic K, Gkouskou K, Trougakos IP, Kolettas E, Georgakilas AG, Volarevic S, Eliopoulos AG, Zannis-Hadjopoulos M, et al. Cdc6 expression represses E-cadherin transcription and activates adjacent replication origins. J Cell Biol. 2011; 195(7):1123-1140.
15. Amatruda JF, Shepard JL, Stern HM and Zon LI. Zebrafish as a cancer model system. Cancer Cell. 2002; 1(3):229-231.

16. Feitsma $\mathrm{H}$ and Cuppen E. Zebrafish as a cancer model. Mol Cancer Res. 2008; 6(5):685-694.

17. Stoletov K and Klemke R. Catch of the day: zebrafish as a human cancer model. Oncogene. 2008; 27(33):4509-4520.

18. Berghmans S, Murphey RD, Wienholds E, Neuberg D, Kutok JL, Fletcher CD, Morris JP, Liu TX, Schulte-Merker S, Kanki JP, Plasterk R, Zon LI and Look AT. tp53 mutant zebrafish develop malignant peripheral nerve sheath tumors. Proc Natl Acad Sci U S A. 2005; 102(2):407-412.

19. Faucherre A, Taylor GS, Overvoorde J, Dixon JE and Hertog J. Zebrafish pten genes have overlapping and non-redundant functions in tumorigenesis and embryonic development. Oncogene. 2008; 27(8):1079-1086.

20. Haramis AP, Hurlstone A, van der Velden Y, Begthel $\mathrm{H}$, van den Born $\mathrm{M}$, Offerhaus GJ and Clevers HC. Adenomatous polyposis coli-deficient zebrafish are susceptible to digestive tract neoplasia. EMBO Rep. 2006; 7(4):444-449.

21. Liu TX, Howlett NG, Deng M, Langenau DM, Hsu K, Rhodes J, Kanki JP, D'Andrea AD and Look AT. Knockdown of zebrafish Fancd2 causes developmental abnormalities via p53-dependent apoptosis. Dev Cell. 2003; 5(6):903-914.

22. Ju B, Spitsbergen J, Eden CJ, Taylor MR and Chen W. Co-activation of hedgehog and AKT pathways promote tumorigenesis in zebrafish. Mol Cancer. 2009; 8:40.

23. Davison JM, Woo Park S, Rhee JM and Leach SD. Characterization of Kras-mediated pancreatic tumorigenesis in zebrafish. Methods Enzymol. 2008; 438:391-417.

24. Nguyen AT, Emelyanov A, Koh CH, Spitsbergen JM, Parinov $\mathrm{S}$ and Gong Z. An inducible krasV12 transgenic zebrafish model for liver tumorigenesis and chemical drug screening. Dis Model Mech. 2012; 5(1):63-72.

25. Vafa O, Wade M, Kern S, Beeche M, Pandita TK, Hampton GM and Wahl GM. c-Myc can induce DNA damage, increase reactive oxygen species, and mitigate p53 function: a mechanism for oncogene-induced genetic instability. Mol Cell. 2002; 9(5):1031-1044.

26. Liu E, Lee AY, Chiba T, Olson E, Sun P and Wu X. The ATR-mediated $\mathrm{S}$ phase checkpoint prevents rereplication in mammalian cells when licensing control is disrupted. J Cell Biol. 2007; 179(4):643-657.

27. Iwatsuki M, Mimori K, Yokobori T, Ishi H, Beppu T, Nakamori S, Baba H and Mori M. Epithelial-mesenchymal transition in cancer development and its clinical significance. Cancer Sci. 2010; 101(2):293-299.

28. Michailidou C, Jones M, Walker P, Kamarashev J, Kelly A and Hurlstone AF. Dissecting the roles of Raf- and PI3K-signalling pathways in melanoma formation and progression in a zebrafish model. Dis Model Mech. 2009; 2(7-8):399-411.

29. Patel JH, Loboda AP, Showe MK, Showe LC and 
McMahon SB. Analysis of genomic targets reveals complex functions of MYC. Nat Rev Cancer. 2004; 4(7):562-568.

30. Dang CV, O’Donnell KA, Zeller KI, Nguyen T, Osthus RC and Li F. The c-Myc target gene network. Semin Cancer Biol. 2006; 16(4):253-264.

31. Felsher DW and Bishop JM. Transient excess of MYC activity can elicit genomic instability and tumorigenesis. Proc Natl Acad Sci U S A. 1999; 96(7):3940-3944.

32. Sankar N, Kadeppagari RK and Thimmapaya B. c-Mycinduced aberrant DNA synthesis and activation of DNA damage response in p300 knockdown cells. J Biol Chem. 2009; 284(22):15193-15205.

33. Robinson K, Asawachaicharn N, Galloway DA and Grandori C. c-Myc accelerates S-Phase and requires WRN to avoid replication stress. PLoS One. 2009; 4(6):e5951.

34. Karlsson A, Deb-Basu D, Cherry A, Turner S, Ford J and Felsher DW. Defective double-strand DNA break repair and chromosomal translocations by MYC overexpression. Proc Natl Acad Sci U S A. 2003; 100(17):9974-9979.

35. Di Paola D and Zannis-Hadjopoulos M. Comparative analysis of pre-replication complex proteins in transformed and normal cells. J Cell Biochem. 2012; 113(4):1333-1347.

36. Chen CF, Chu CY, Chen TH, Lee SJ, Shen CN and Hsiao CD. Establishment of a transgenic zebrafish line for superficial skin ablation and functional validation of apoptosis modulators in vivo. PLoS One. 2011; 6(5):e20654.

37. Izumi N, Yamashita A, Hirano H and Ohno S. Heat shock protein 90 regulates phosphatidylinositol 3-kinase-related protein kinase family proteins together with the RUVBL1/2 and Tel2-containing co-factor complex. Cancer science. 2012; 103(1):50-57.

38. Luo J, Deng ZL, Luo X, Tang N, Song WX, Chen J, Sharff KA, Luu HH, Haydon RC, Kinzler KW, Vogelstein B and He TC. A protocol for rapid generation of recombinant adenoviruses using the AdEasy system. Nat Protoc. 2007; 2(5):1236-1247.

39. Lee AY, Chiba T, Truong LN, Cheng AN, Do J, Cho MJ, Chen $\mathrm{L}$ and $\mathrm{Wu} \mathrm{X}$. Dbf4 Is Direct Downstream Target of Ataxia Telangiectasia Mutated (ATM) and Ataxia Telangiectasia and Rad3-related (ATR) Protein to Regulate Intra-S-phase Checkpoint. J Biol Chem. 2012; 287(4):25312543.

40. Vaziri C, Saxena S, Jeon Y, Lee C, Murata K, Machida Y, Wagle N, Hwang DS and Dutta A. A p53-dependent checkpoint pathway prevents rereplication. Mol Cell. 2003; 11(4):997-1008. 